\title{
Erratum to: Reliability of magnetic resonance imaging measurements of the cross-sectional area of the muscle contractile and non-contractile components
}

\author{
Olivier Gille • Mathieu-Panchoa de Sèze • \\ Patrick Guérin · Erwan Jolivet · Jean-Marc Vital • \\ Wafa Skalli
}

Published online: 7 July 2011

(C) Springer-Verlag 2011

\section{Erratum to: Surg Radiol Anat \\ DOI 10.1007/s00276-011-0825-7}

In the original publication of this article there is a change in corresponding author's name and the order of the authors. Also one of the co-author Jean-Marc Vital was missed in the author group. The correct author group and their affiliations are given below:

Olivier Gille · Mathieu-Panchoa de Sèze · Patrick Guérin · Erwan Jolivet · Jean-Marc Vital · Wafa Skalli

M.-P. de Sèze

EA 4136 Handicap et système nerveux-IFR8-IFR25, Service de Médecine Physique et de Réadaptation, Pôle Neurosciences Cliniques, Université Victor Segalen Bordeaux 2, CHU Bordeaux Pellegrin, 33076 Bordeaux cedex, France

O. Gille and M.-P. de Sèze contributed equally to this work.

The online version of the original article can be found under doi:10.1007/s00276-011-0825-7.

M.-P. de Sèze

EA 4136 Handicap et système nerveux-IFR8-IFR25, Service de Médecine Physique et de Réadaptation, Pôle Neurosciences

Cliniques, Université Victor Segalen Bordeaux 2,

CHU Bordeaux Pellegrin, 33076 Bordeaux cedex, France

O. Gille $(\bowtie) \cdot$ P. Guérin · J.-M. Vital

Spinal Unit, CHU Bordeaux Pellegrin,

33076 Bordeaux cedex, France

e-mail: olivier.gille@chu-bordeaux.fr

O. Gille · E. Jolivet · W. Skalli

Laboratoire de Biomécanique, Arts et Métiers ParisTech,

151 bd de l'Hôpital, 75013 Paris, France
O. Gille $(\bowtie) \cdot$ P. Guérin · J.-M. Vital

Spinal Unit, CHU Bordeaux Pellegrin, 33076 Bordeaux cedex, France

O. Gille · E. Jolivet · W. Skalli

Laboratoire de Biomécanique, Arts et Métiers ParisTech, 151 bd de l'Hôpital, 75013 Paris, France 